\title{
Factors Affecting Metabolic Syndrome in Individuals With Chronic Spinal Cord Injury
}

\author{
Ji Won Shin, MD, Tayeon Kim, MD, Bum-Suk Lee, MD, MPH, Onyoo Kim, MD
}

Department of Rehabilitation Medicine, National Rehabilitation Center, Seoul, Korea

\begin{abstract}
Objective To assess the validity of different anthropometric measures (waist circumference [WC], body mass index [BMI], and percentage body fat) in diagnosing metabolic syndrome (MetS) among individuals with SCI and provides preliminary data for future studies in setting obesity cutoff values for this population.

Methods This was a single-center retrospective cohort study. Sample information, anthropometric measures, and MetS variables of 157 individuals with chronic SCI were collected from an electronic medical records database.

Results Increasing age (odds ratio $[\mathrm{OR}]=1.040, \mathrm{p}=0.016)$ and lower neurological level of injury $(\mathrm{OR}=1.059$, $\mathrm{p}=0.046)$ were risk factors for MetS. Male BMI $(\mathrm{r}=0.380, \mathrm{p}<0.001)$ and male WC $(\mathrm{r}=0.346, \mathrm{p}<0.001)$ were positively correlated with the number of MetS subfactors. Individuals with non-obese WC, excluding central obesity, were associated with having no MetS subfactors ( $\mathrm{p}=0.005)$, and individuals with obese WC were associated with one or more subfactors $(\mathrm{p}=0.005)$. BMI was associated with MetS diagnosis (area under the curve $=0.765, \mathrm{p}<0.001$ ), with the calculated cutoff value for BMI being $22.8 \mathrm{~kg} / \mathrm{m}^{2}$.

Conclusion This study calls for a stricter BMI cutoff for individuals with SCI in diagnosing MetS and warrants a large population-based study to define central obesity according to sex and ethnicity.
\end{abstract}

Keywords Spinal cord injuries, Metabolic syndrome, Obesity

\section{INTRODUCTION}

Individuals with spinal cord injury (SCI), compared with the general population, have a higher risk of morbidity and mortality, with cardiovascular disease (CVD) being the most common cause [1]. In addition, metabolic syndrome (MetS), a component of central obesity, hypertension, hyperglycemia, and dyslipidemia, is associated with a higher CVD risk [2]. After SCI, individuals undergo drastic changes in terms of decreased physical activity, decreased muscle mass, and lower metabolism. These changes result in an increased prevalence of obesity, linked to an increased risk of MetS [3]. Thus, an accurate definition of obesity is vital in identifying individuals at risk of MetS for both weight assessment and management.

Received August 9, 2021; Revised November 1, 2021; Accepted December 13, 2021; Published online February 28, 2022

Department of Rehabilitation Medicine, National Rehabilitation Center, 58 Samgaksan-ro, Gangbuk-gu, Seoul 01022, Korea. Tel: +82-02-901-1883, E-mail: ohnew33@korea.kr

ORCID: Ji Won Shin (https://orcid.org/0000-0002-6227-3413); Tayeon Kim (https://orcid.org/0000-0002-8826-825X); Bum-Suk Lee (https://orcid. org/0000-0002-0506-0403); Onyoo Kim (https://orcid.org/0000-0001-9517-2240).

(c) This is an open-access article distributed under the terms of the Creative Commons Attribution Non-Commercial License (http://creativecommons.org/ licenses/by-nc/4.0) which permits unrestricted noncommercial use, distribution, and reproduction in any medium, provided the original work is properly cited. Copyright $\odot 2022$ by Korean Academy of Rehabilitation Medicine 
The emphasis on finding the optimal cutoff value to define obesity derives from the fact that central obesity is the most prevalent feature of MetS [4]. This statement is supported by a rapid parallel increase in the prevalence of overweight, obesity, type 2 diabetes, and MetS [5]. Furthermore, although there are cases of metabolically benign obesity, which is not accompanied by insulin resistance and atherosclerosis [6], the vast majority are insulin resistant. In particular, individuals with central accumulation of body fat are at a higher risk of insulin resistance and atherogenic abnormalities $[7,8]$, whereas the risk is less for those with body fat distribution in the periphery [9]. Thus, due to the increasing recognition of central obesity as the most common feature of MetS, in this study, the diagnosis of MetS has been used as a surrogate for obesity.

After SCI, the percentage of visceral adipose tissue (VAT) quantified by computed tomography (CT) at the umbilical and L4-L5 levels was found to be $34 \%$ [10] and $45 \%$ [11], respectively, which is higher in individuals with SCI. Even after considering the decreased abdominal muscle tone, which results in measurement discrepancies in the supine versus seated position [12], Ravensbergen et al. [13] showed that individuals with SCI had $42 \%$ more VAT at the same WC than the general population. They evaluated the relationship between different anthropometric measures and the abdominal fat percentage, cardiovascular, metabolic risk factors, and the Framingham risk score among 27 individuals with SCI and suggested a waist circumference (WC) $\geq 94 \mathrm{~cm}$ as the optimal cutoff value. Sumrell et al. [12] conducted a study on 22 men with SCI to find the optimal WC cutoff value of $86.5 \mathrm{~cm}$ in the supine position. Inayama et al. [14] identified $\mathrm{WC} \geq 81.3 \mathrm{~cm}$ to correspond with a visceral fat area of $\geq 100 \mathrm{~cm}^{2}$ in 74 Japanese men with SCI in the supine position. These findings suggest that the cutoff point for WC should be lower in individuals with SCI.

The three commonly used anthropometric measures for identifying obesity are percentage body fat (PBF), body mass index (BMI), and WC. For these measures, the cutoff values for obesity were defined in the general population. However, when extrapolated to individuals with SCI, these cutoffs values for obesity run the risk of underdiagnosing MetS. The arbitrary nature of cutoffs for obesity was introduced by the International Diabetes Federation (IDF), proposing ethnically appropriate
WC to be considered in diagnosing MetS [15]. However, the reevaluation of cutoff values in individuals with SCI, prone to body composition and metabolic changes, has been under-addressed.

Thus, the purpose of this study was (1) to identify the risk factors of MetS, (2) to determine the correlation and association between different anthropometric measures and the number of MetS subfactors, and (3) to provide preliminary data for future studies in setting obesity cutoff values for Korean patients with SCI.

\section{MATERIALS AND METHODS}

Approval of the Institutional Review Board at the National Rehabilitation Center was obtained before conducting the study (No. NRC-2020-02-021), and the study was performed following the Declaration of Helsinki of the World Medical Association.

\section{Patients}

A total of 166 Korean patients with chronic SCI (time since injury $\geq 1$ year) were admitted to a single center in Seoul, Korea, from February to December 2019, for a 3-day comprehensive checkup program, which included a urodynamic study, blood and urine tests, serial vital sign check, and anthropometric measurements. Of the 166 patients, nine patients were excluded, as eight individuals had unknown SCI onset dates, and one patient had missing PBF data. Thus, 157 patients were enrolled in this study. In addition, retrospective sample information, anthropometric measures, and MetS variables were collected from electronic medical records.

We collected information regarding age, sex, time since injury, cause of injury, lesion severity (i.e., paraplegia, tetraplegia), the American Spinal Injury Association impairment scale (AIS) scores, and the level of injury severity. The motor complete injury was defined as AIS level $\mathrm{A}$ or $\mathrm{B}$, and the motor incomplete injury was defined as AIS level C or D [16]. Detailed clinical and neurological examinations were based on the International Standards for Neurological Classification of Spinal Cord Injury (ISNCSCI).

\section{Anthropometric measurements}

Anthropometric measurements of height $(\mathrm{cm})$, weight (kg), WC, and PBF were performed. Height was mea- 
sured from the vertex level to the foot to the nearest $1 \mathrm{~cm}$. Weight was measured using a weighing scale to the nearest $0.1 \mathrm{~kg}$, and for patients who were wheelchair-bound, the weight of the wheelchair was deducted from the sum of wheelchair and patient weight. The WC was measured at the narrowest level (i.e., umbilical level) at the end of normal expiration in the supine position. The PBF was measured through bioelectrical impedance analysis using InBodyS10 (Biospace Co. Ltd, Seoul, Korea). The BMI was calculated using the formula, BMI $=\mathrm{kg} / \mathrm{m}^{2}$, where $\mathrm{kg}$ is a person's weight in kilograms and $\mathrm{m}^{2}$ is their height in meters squared.

\section{Obesity classification}

The World Health Organization (WHO) classification of BMI $\geq 30 \mathrm{~kg} / \mathrm{m}^{2}$ was used to define obesity. Although the National Institutes of Health classify individuals with WC $>102 \mathrm{~cm}$ for men and $>88 \mathrm{~cm}$ for women as obese, the ethnically appropriate cutoff levels for Korean adults, WC $>90 \mathrm{~cm}$ for men and $>85 \mathrm{~cm}$ for women, were adopted in this study [15]. We used the most recent 2009 guidelines from the American Society of Bariatric Physicians (ASBP), an American Medical Association (AMA) specialty board, PBF $\geq 25 \%$ for men and $\geq 30 \%$ for women, to define obesity.

\section{MetS classification}

The American Heart Association and the National Heart, Lung, and Blood Institute (AHA/NHLBI) [17] and the National Cholesterol Education Program Adult Treatment Panel III (NCEP-ATP III) [18] classifications were compared to diagnose MetS. The NCEP-ATP III criteria are easily applicable to clinical settings and large-scale epidemiological studies [19]. Recently, the AHA/NHLBI expanded the NCEP-ATP III criteria to include those who also received drug therapy for a more inclusive diagnosis [20]. The MetS classifications used are summarized in Supplementary Table S1. Diagnosis of MetS was set with three or more subfactors present. The WC cutoff value was substituted for Korean adults' ethnically appropriate cutoff value.

Triglyceride (TG), high-density lipoprotein (HDL) cholesterol, and fasting glucose levels were measured through blood tests on the second day of admission, after a 12-hour fasting period overnight. In addition, blood pressure was averaged from measurements taken in the morning on the second and third day of admission.

\section{Statistical analysis}

Descriptive statistics were used to summarize demographic and lesion characteristics. Binomial logistic regression was used to evaluate risk factors for MetS. The Pearson correlation test was used to determine the correlation between different anthropometric measures and the number of MetS subfactors in male and female patients. To further quantitatively analyze the relationship between obesity according to different anthropometric measures and the number of MetS subfactors, we performed cross-tabulation with linear-by-linear association chi-square test. The area under the ROC curve (AUC) was used to determine the cutoff value for different anthropometric measures and their validity, respectively, relative to the MetS diagnosis. We used the SPSS version 21.0 (IBM SPSS, Armonk, NY, USA) for statistical analysis, and a statistically significant difference was considered at $\mathrm{p}<0.05$.

\section{RESULTS}

\section{Characteristics of the sample}

The demographic and injury characteristics of the sample, summarized by descriptive statistics of the sample, are displayed in Table 1. The participants were predominantly men $(n=110 ; 70.1 \%)$. The mean age at the time of the study was 49 years (standard deviation $[\mathrm{SD}]=12.3$ ). The mean time since injury was 12 years $(\mathrm{SD}=7.9)$. Most injuries were due to trauma ( $\mathrm{n}=133 ; 84.7 \%)$. More than half resulted in paraplegia $(\mathrm{n}=81 ; 51.6 \%)$, and AIS-A/ complete injury ( $\mathrm{n}=99 ; 63.1 \%)$ was the most common severity of the injury.

\section{MetS prevalence}

In NCEP-ATP III criteria, the prevalence of MetS was $23.6 \%(n=37)$, consisting of male $(n=31,28.2 \%)$ and female ( $\mathrm{n}=6,12.8 \%)$. In AHA/NHLIB criteria, the prevalence of MetS was $38.9 \%(n=61)$, consisting of men $(n=42$, $38.2 \%)$ and female $(n=19,40.4 \%)$. All individuals with NCEP-ATP III-classified MetS were included in the group with AHA/NHLBI-classified MetS.

\section{MetS risk factors}

Binomial logistic regression was used to evaluate risk factors for the diagnosis of MetS. Among the indepen- 
dent variables, increasing age (odds ratio $[\mathrm{OR}]=1.040$; 95\% confidence interval [CI], 1.007-1.073; $\mathrm{p}=0.016)$ and lower neurological level of injury (NLI) (OR=1.059; 95\% CI, 1.001-1.119; $\mathrm{p}=0.046$ ) were significant risk factors for MetS (Table 2).

Correlation between the number of MetS subfactors and different anthropometric measures

Pearson correlation test was used to assess the correla-

Table 1. Demographic and lesion characteristics

\begin{tabular}{|lc|}
\hline \multicolumn{1}{c}{ Characteristic } & Value \\
\hline Age $(\mathrm{yr})$ & $49.3 \pm 12.3$ \\
\hline Sex & \\
\hline Male & $110(70.1)$ \\
\hline Female & $47(29.9)$ \\
\hline Time since injury (yr) & $12.0 \pm 7.9$ \\
\hline Cause of spinal cord injury & \\
\hline Traumatic & $133(84.7)$ \\
\hline Non-traumatic & $24(15.3)$ \\
\hline Lesion severity & \\
\hline Paraplegia & $81(51.6)$ \\
\hline Tetraplegia & $76(48.4)$ \\
\hline AIS grade & \\
\hline A & $99(63.1)$ \\
\hline B & $20(12.7)$ \\
\hline C & $18(11.5)$ \\
\hline D & $20(12.7)$ \\
\hline
\end{tabular}

Values are presented as mean \pm standard deviation or number (\%).

AIS, American Spinal Injury Association impairment scale. tion between different anthropometric measures and the number of MetS subfactors, that is, elevated fasting glucose and TG, and reduced HDL-cholesterol levels, elevated values of the $\mathrm{BP}$ and $\mathrm{WC}$, or whether on drug treatment for each subfactor, depending on the sex (Table $3)$. The BMI $(\mathrm{r}=0.380, \mathrm{p}<0.001)$ and the WC $(\mathrm{r}=0.346$, $\mathrm{p}<0.001)$ in men were significantly positively correlated with the number of MetS subfactors.

\section{Association between the number of MetS subfactors and obesity}

Further quantitative analysis of the linear relationship between the number of MetS subfactors and obesity according to different anthropometric measures was performed using cross-tabulation with a linear-bylinear association chi-square test (Table 4). As the WC is a dependent variable of the AHA/NHLBI criteria, the subfactor "elevated WC" was excluded from the analysis. Non-obese values for the WC were significantly associated with having no MetS subfactors (linear-by-linear association $=7.872, p=0.005$ ). Increased $W C$ value was significantly associated with having one or more MetS subfactors (linear-by-linear association $=7.872, \mathrm{p}=0.005$ ).

\section{Cutoff values}

The AUC were used to determine the cutoff value for anthropometric measures and their validity, respectively, relative to the diagnosis of MetS (Table 5). Assuming that AHA/NHLBI-classified MetS in individuals with SCI translated to a diagnosis of MetS, the WC was excluded from the anthropometric measures, as it was a dependent variable of the AHA/NHLBI classification. The BMI

Table 2. Binomial logistic regression analysis of metabolic syndrome risk factors

\begin{tabular}{|lccc}
\hline & OR & $\mathbf{9 5 \%}$ CI & p-value \\
\hline Sex (female) & 0.854 & $0.395-1.847$ & 0.689 \\
\hline Age (increasing) & 1.040 & $1.007-1.073$ & $0.016^{*}$ \\
\hline BMI (obese, BMI $\geq 30$ ) & 1.244 & $0.205-7.552$ & 0.813 \\
\hline PBF (obese, male $\geq 25 \%$, female $\geq 30 \%$ ) & 1.275 & $0.432-3.757$ & 0.660 \\
\hline Time since injury (increasing) & 0.980 & $0.936-1.025$ & 0.378 \\
\hline Cause of injury (traumatic) & 0.440 & $0.155-1.245$ & 0.122 \\
\hline NLI (lower) & 1.059 & $1.001-1.119$ & $0.046^{*}$ \\
\hline Severity of injury (motor incomplete) & 0.816 & $0.324-2.057$ & 0.666 \\
\hline
\end{tabular}

BMI, body mass index; PBF, percentage body fat; NLI, neurological level of injury; OR, odds ratio; CI, confidence interval.

${ }^{*} \mathrm{p}<0.05$. 
Table 3. Pearson correlation coefficients between the number of metabolic syndrome subfactors and anthropometric measures

\begin{tabular}{ccccc}
\hline & \multicolumn{3}{c}{ Male } & \multicolumn{3}{c}{ Female } \\
\cline { 2 - 3 } \cline { 5 - 5 } & $\mathbf{r}$ & p-value & r & p-value \\
\hline WC & 0.380 & $<0.001^{* * *}$ & 0.213 & 0.151 \\
BMI & 0.346 & $<0.001^{* * *}$ & 0.234 & 0.113 \\
PBF & 0.145 & 0.130 & 0.016 & 0.916 \\
\hline
\end{tabular}

WC, waist circumference; BMI, body mass index; PBF, percentage body fat; r, Pearson correlation coefficient. ${ }^{* * *} \mathrm{p}<0.001$.

Table 4. Cross-tabulation between the number of metabolic syndrome (MetS) subfactors and obesity

\begin{tabular}{|c|c|c|c|c|c|c|}
\hline \multirow{2}{*}{$\begin{array}{l}\text { Number of MetS } \\
\text { subfactors }\end{array}$} & \multicolumn{2}{|c|}{ WC } & \multicolumn{2}{|c|}{ BMI } & \multicolumn{2}{|c|}{ PBF } \\
\hline & Non-obese & Obese & Non-obese & Obese & Non-obese & Obese \\
\hline 0 & $24(27.0)$ & $2(2.9)$ & $26(17.2)$ & $0(0)$ & 7 (33.3) & $19(14.0)$ \\
\hline 1 & $21(23.6)$ & $22(32.4)$ & $40(26.5)$ & $3(50.0)$ & $5(23.8)$ & $38(27.9)$ \\
\hline 2 & $27(30.3)$ & $25(36.8)$ & $52(34.4)$ & $0(0)$ & $2(9.5)$ & $50(36.8)$ \\
\hline 3 & $12(13.5)$ & $13(19.1)$ & $23(15.2)$ & $2(33.3)$ & $5(23.8)$ & $20(14.7)$ \\
\hline 4 & $5(5.6)$ & $6(8.8)$ & $10(6.6)$ & $1(16.7)$ & $2(9.5)$ & $9(6.6)$ \\
\hline p-value & \multicolumn{2}{|c|}{$7.872(0.005)^{* *}$} & \multicolumn{2}{|c|}{$1.079(0.299)$} & \multicolumn{2}{|c|}{$0.546(0.460)$} \\
\hline
\end{tabular}

WC, waist circumference; BMI, body mass index; PBF, percentage body fat.

Cutoff values: WC (male $\geq 90 \mathrm{~cm}$, female $\geq 85 \mathrm{~cm}$ ), BMI (male $\geq 30 \mathrm{~kg} / \mathrm{m}^{2}$, female $\geq 30 \mathrm{~kg} / \mathrm{m}^{2}$ ), $\mathrm{PBF}$ (male $\geq 25 \%$, female $\geq 30 \%$ ).

${ }^{* *} \mathrm{p}<0.01$ by linear-by-linear association test.

Table 5. Cutoff values of different anthropometric measurements relative to the diagnosis of metabolic syndrome

\begin{tabular}{lccccc}
\hline & Cutoff value & Sensitivity & Specificity & AUC (95\% CI) & p-value \\
\hline BMI & 22.835 & 0.721 & 0.729 & $0.765(0.689-0.842)$ & $<0.001^{* * *}$ \\
\hline PBF & & & & & \\
Male & 37.650 & 0.595 & 0.588 & $0.603(0.497-0.710)$ & 0.690 \\
Female & 39.550 & 0.632 & 0.643 & $0.565(0.386-0.744)$ & 0.455 \\
\hline
\end{tabular}

BMI, body mass index; PBF, percentage body fat; AUC, area under the curve; CI, confidence interval.

${ }^{* * *} \mathrm{p}<0.001$.

(AUC=0.765; 95\% CI, 0.689-0.842, $\mathrm{p}<0.001$ ) was significantly associated with diagnosis of MetS, with a cutoff value of $22.8 \mathrm{~kg} / \mathrm{m}^{2}$ (sensitivity=72.1\%, specificity=72.9\%).

\section{DISCUSSION}

The demographic data collected from our study showed a male-to-female ratio of $68.1 \%$ to $31.9 \%$, in sync with the National Spinal Cord Injury Statistical Center (NSCISC) database of 33,406 people with SCI between 2015 and 2018. There, the male-to-female ratio was $78 \%$ to $22 \%$. The mean age at the time of the study was $49.3 \pm 12.3$ years, which is reflective of the aging population with SCI, with the average age at injury increasing from 29 to 43 years from the 1970s to 2018, according to the NSCISC database. Though the NSCISC database showed a greater prevalence of tetraplegia (59.9\%) than paraplegia (39.5\%), patients who visited our center for a 3-day checkup program were $51.6 \%$ paraplegic and $48.4 \%$ tetraplegic. This difference could be because most of the patients admitted to the 3-day checkup program were homedischarged, a more common practice among patients with paraplegia [21]. Our center had a higher proportion of complete SCI $(n=99 ; 63.1 \%)$ to incomplete SCI $(n=58$; 
$36.9 \%$ ) individuals, than $31.9 \%$ to $67.5 \%$ in the NSCISC database. The higher prevalence of individuals with complete SCI admitted to the 3-day checkup program could be because the program focuses on neurogenic bladder management and urodynamic study. Individuals with complete SCI have more difficulty managing the bladder drainage method [22].

Four definitions of MetS given by the IDF, AHA/NHLBI, NCEP-ATP III, and WHO criteria are commonly used in studies and clinical settings. Ma et al. [20] suggested that MetS defined by the IDF and AHA/NHLBI classifications presented with significantly higher carotid thickness. Skilton et al. [23] proposed that MetS diagnosed following the AHA/NHLBI classification was the strongest predictor of carotid atherosclerosis among males. At present, there are still ongoing debates regarding the optimal classification for MetS and the validity of extrapolating the same standards to individuals with SCI [24]. This study included all NCEP-ATP III-classified participants with MetS $(\mathrm{n}=37 ; 23.6 \%)$ in the group with AHA/NHLBI-classified MetS ( $n=61 ; 38.9 \%)$. Thus, the AHA/NHLBI criteria were deemed more inclusive in the diagnosis of MetS, which resulted in our decision to follow the AHA/NHLBI guidelines for this study.

Hirode and Wong [25] evaluated the trends in MetS prevalence among 17,048 adults (aged $\geq 20$ years) from the general United States population using National Health and Nutrition Examination Survey data from 2011 to 2016. Among Asian adults, the MetS prevalence increased significantly from $19.9 \%$ to $26.2 \%$ over the study period (2011-2016), with a total mean prevalence of $23.2 \%$. This study showed a similar prevalence of NCEP-ATPIII-classified MetS individuals ( $\mathrm{n}=37 ; 23.6 \%)$. In addition, Gater et al. [26] conducted a cross-sectional study of 473 veterans with SCI. NCEP-ATP III-classified MetS was diagnosed in $22.9 \%$ of the veterans using $\mathrm{BMI} \geq 30 \mathrm{~kg} / \mathrm{m}^{2}$ as the cutoff value for obesity. These studies show a similar prevalence of MetS between the general population and individuals with SCI. However, Maruyama et al. [10] found that $43 \%$ of 44 men with SCI were classified with MetS according to the NCEP-ATP III definition, using a WC cutoff value, which corresponded to the obesity cutoff point for visceral fat adiposity as $100 \mathrm{~cm}$ [27], defined by the Japanese Society for the Study of Obesity, as $85 \mathrm{~cm}$ for men and $90 \mathrm{~cm}$ for women. The higher prevalence of MetS in Japanese men with SCI than that in general Japanese workers $(19.5 \%$ in men and 7.9\% in women) [28] differed from the prevalence of MetS in this study. Although the WC cutoff value for Japanese men is $5 \mathrm{~cm}$ lower than that of Korean men, the greater prevalence of MetS in the SCI population than the general population should be considered. It calls for a reevaluation of the WC cutoff values.

Individuals with tetraplegia are known to have a higher risk of insulin resistance [29], lower metabolism, and lower daily activity, which increases the risk of MetS compared with those with paraplegia [30]. However, our finding points toward lower NLI as a risk factor, partly because individuals with paraplegia have increased independence in lifestyle and eating habits [31]. This phenomenon has also been reported in other studies, where individuals with paraplegia had higher average BMI, WC, biceps, and triceps skin fold thickness measures than those with tetraplegia [32]. Regarding the "elevated BP levels", a MetS subfactor, individuals with tetraplegia are predisposed to impaired control of the autonomic nervous system, which can lead to hypotension, bradycardia, and events of uncontrolled hypertension [33]. Since BP levels are generally taken from the resting state, individuals with tetraplegia are subject to being omitted from the "elevated BP levels" subfactor. However, it is known that autonomic dysregulation due to SCI is associated with a cardiovascular risk [34], which might be misrepresented by the current MetS classification defined for the general population.

While the BMI and WC values in men both showed a significant positive correlation with the number of MetS subfactors, the WC was the single anthropometric measure that was significantly associated with the number of the MetS subfactors, excluding "elevated WC values". Non-obese patients defined by their WC values (obese: men $\geq 90 \mathrm{~cm}$, women $\geq 85 \mathrm{~cm}$ ) had a significantly higher probability of having no subfactors by $27.0 \%$ compared with $2.9 \%$ of those classified as obese. Individuals classified as obese by their WC values had an increased chance of having one or more subfactors in every number of subfactors (Table 4). This suggests that obesity defined by the WC values is more closely associated with subfactors constituting MetS than the BMI. Inayama et al. [14] reported that although WC and BMI were both significantly associated with the visceral fat area, WC was more accurate than BMI. This result is in line with other studies favoring WC to define obesity in individuals with SCI, 
as BMI fails to distinguish between fat and fat-free mass and is an insufficient biomarker of abdominal adiposity [5]. Other studies have underlined the reliability of the WC measurement as a simple method to determine abdominal adiposity and its clinically applicable nature, presenting strong associations with all-cause $[35,36]$ and cardiovascular mortality $[37,38]$.

Unfortunately, as the diagnosis of MetS was used in this study as a surrogate for obesity, the cutoff value for the WC could not be obtained due to its inclusion in the AHA/NHLBI criteria. However, the BMI, which is an independent variable of the AHA/NHLBI classification of MetS, was significantly associated with MetS diagnosis (AUC=0.765; 95\% CI, 0.689-0.842; $\mathrm{p}<0.001$ ) with 22.835 $\mathrm{kg} / \mathrm{m}^{2}$ (sensitivity $=72.1 \%$, specificity $=72.9 \%$ ) as a suggested cutoff value. Likewise, Laughton et al. [39] suggested that the BMI cutoff for obesity should be lowered to $22 \mathrm{~kg} / \mathrm{m}^{2}$ based on the percentage of fat mass and the C-reactive protein levels in a study of 77 individuals with chronic SCI. Inayama et al. [14] also identified $22.5 \mathrm{~kg} / \mathrm{m}^{2}$ as a BMI cutoff value corresponding to a visceral fat area of $\geq 100 \mathrm{~cm}^{2}$. Although the BMI has limitations in reflecting body fat, it is more reliable than weight alone and constitutes readily available anthropometric measures, such as weight and height. The suggested cutoff value for BMI stresses the need to define obesity in the SCI population properly.

This study had several limitations. First, the sample size was relatively small. In addition, the sample was from a distinctive 3-day checkup program focusing on evaluating and managing neurogenic bladder in individuals with SCI, which led to a disproportionate deviation toward those with paraplegia and complete injury. Second, the patients were recommended for annual follow-ups, and since their overall well-being was being evaluated and managed regularly, the sample from the program might have had fewer MetS. Third, the PBF was measured using the bioelectrical impedance analysis, which is subject to validation in reading because individuals with SCI show a predisposition [40]. Fourth, the cutoff values used for defining obesity were based on the general population, which might have underestimated the prevalence of the MetS when applied to individuals with SCI. Finally, the use of the MetS as a surrogate for central obesity has its limitations; although central obesity is associated with atherogenic and insulin-related abnormalities, it is not a mandatory criterion of the AHA/NHLBI classification. Furthermore, it is unknown whether it causes or is correlated to MetS [4]. Although the WC was the single anthropometric measure with a significant positive correlation and association with the number of MetS subfactors, the cutoff value between the WC and MetS diagnosis could not be found due to their codependent nature. The cutoff value was investigated between different anthropometric measures and the MetS diagnosis using a classification made for the general population. Thus, the validity of the subfactors of the MetS itself is questionable when subjected to SCI, as they are prone to autonomic dysregulation. Lastly, since there is no consensus for SCI-specific WC cutoff values, we were predisposed to use the ethnically appropriate Korean adult WC cutoff values. However, in SCI conditions, using disease-specific cutoff values could be more accurate than ethnic-specific WC cutoff values, leading to potentially different observations in our study. Thus, further large-scale studies are required to determine the Korean SCI-specific cutoff values for the WC and BMI using dual-energy X-ray absorptiometry or other imaging methods that provide VAT quantification.

This study aimed to determine the optimal anthropometric measure and its cutoff value for individuals with SCI in diagnosing the MetS. Among the different anthropometric measures, the WC was the sole measure that had both a significant positive correlation and, based on the obesity cutoffs provided for the general population, a significant linear relationship between the number of the MetS subfactors and obesity. Although the cutoff value for WC could not be evaluated in this study, the results point to establishing stricter BMI cutoff values for individuals with SCI than for the general population in diagnosing the MetS. In addition, they warrant a large population-based study to redefine the cutoff value for central obesity in individuals with SCI according to sex and ethnicity.

\section{CONFLICT OF INTEREST}

No potential conflict of interest relevant to this article was reported.

\section{AUTHOR CONTRIBUTION}

Conceptualization: Kim O, Kim T. Methodology: Shin 
JW, Kim T, Lee BS, Kim O. Formal analysis: Kim T, Shin JW. Project administration: Kim T, Lee BS, Kim O. Visualization: Kim T, Lee BS, Kim O. Writing - original draft: Kim O, Shin JW. Writing - review and editing: Kim O, Shin JW. Approval of the final manuscript: all authors.

\section{SUPPLEMENTARY MATERIALS}

Supplementary materials can be found via https://doi. org/10.5535/arm.21144.

\section{REFERENCES}

1. Garshick E, Kelley A, Cohen SA, Garrison A, Tun CG, Gagnon D, et al. A prospective assessment of mortality in chronic spinal cord injury. Spinal Cord 2005;43:40816.

2. Torpy JM, Lynm C, Glass RM. The metabolic syndrome. JAMA 2006;295:850.

3. Chen YM, Ho SC, Lam SS, Chan SS. Validity of body mass index and waist circumference in the classification of obesity as compared to percent body fat in Chinese middle-aged women. Int J Obes (Lond) 2006;30:918-25.

4. Despres JP, Lemieux I. Abdominal obesity and metabolic syndrome. Nature 2006;444:881-7.

5. Mokdad AH, Ford ES, Bowman BA, Dietz WH, Vinicor F, Bales VS, et al. Prevalence of obesity, diabetes, and obesity-related health risk factors, 2001. JAMA 2003;289:76-9.

6. Stefan N, Kantartzis K, Machann J, Schick F, Thamer $\mathrm{C}$, Rittig K, et al. Identification and characterization of metabolically benign obesity in humans. Arch Intern Med 2008;168:1609-16.

7. Despres JP, Moorjani S, Lupien PJ, Tremblay A, Nadeau A, Bouchard C. Regional distribution of body fat, plasma lipoproteins, and cardiovascular disease. Arteriosclerosis 1990;10:497-511.

8. Despres JP. Is visceral obesity the cause of the metabolic syndrome? Ann Med 2006;38:52-63.

9. Engin A. The definition and prevalence of obesity and metabolic syndrome. Adv Exp Med Biol 2017;960:1-17.

10. Maruyama Y, Mizuguchi M, Yaginuma T, Kusaka M, Yoshida H, Yokoyama K, et al. Serum leptin, abdominal obesity and the metabolic syndrome in individuals with chronic spinal cord injury. Spinal Cord
2008;46:494-9.

11. Edwards LA, Bugaresti JM, Buchholz AC. Visceral adipose tissue and the ratio of visceral to subcutaneous adipose tissue are greater in adults with than in those without spinal cord injury, despite matching waist circumferences. Am J Clin Nutr 2008;87:600-7.

12. Sumrell RM, Nightingale TE, McCauley LS, Gorgey AS. Anthropometric cutoffs and associations with visceral adiposity and metabolic biomarkers after spinal cord injury. PLoS One 2018;13:e0203049.

13. Ravensbergen HR, Lear SA, Claydon VE. Waist circumference is the best index for obesity-related cardiovascular disease risk in individuals with spinal cord injury. J Neurotrauma 2014;31:292-300.

14. Inayama T, Higuchi $Y$, Tsunoda N, Uchiyama H, Sakuma $\mathrm{H}$. Associations between abdominal visceral fat and surrogate measures of obesity in Japanese men with spinal cord injury. Spinal Cord 2014;52:836-41.

15. Lee SY, Park HS, Kim DJ, Han JH, Kim SM, Cho GJ, et al. Appropriate waist circumference cutoff points for central obesity in Korean adults. Diabetes Res Clin Pract 2007;75:72-80.

16. World Health Organization. International perspectives on spinal cord injury [Internet]. Geneva, Switzerland: World Health Organization; 2013 [cited 2022 Jan 20]. Available from: https://apps.who.int/iris/ handle/10665/94190.

17. Grundy SM, Cleeman JI, Daniels SR, Donato KA, Eckel RH, Franklin BA, et al. Diagnosis and management of the metabolic syndrome: an American Heart Association/National Heart, Lung, and Blood Institute Scientific Statement. Circulation 2005;112:2735-52.

18. National Cholesterol Education Program (NCEP) Expert Panel on Detection, Evaluation, and Treatment of High Blood Cholesterol in Adults (Adult Treatment Panel III). Third report of the National Cholesterol Education Program (NCEP) Expert Panel on Detection, Evaluation, and Treatment of High Blood Cholesterol in Adults (Adult Treatment Panel III) final report. Circulation 2002;106:3143-421.

19. Expert Panel on Detection, Evaluation, and Treatment of High Blood Cholesterol in Adults. Executive summary of the third report of the National Cholesterol Education Program (NCEP) Expert Panel on Detection, Evaluation, and Treatment of High Blood Cholesterol in Adults (Adult Treatment Panel III). JAMA 
2001;285:2486-97.

20. Ma WY, Li HY, Hung CS, Lin MS, Chiu FC, Lin CH, et al. Metabolic syndrome defined by IDF and AHA/ NHLBI correlates better to carotid intima-media thickness than that defined by NCEP ATP III and WHO. Diabetes Res Clin Pract 2009;85:335-41.

21. Eastwood EA, Hagglund KJ, Ragnarsson KT, Gordon WA, Marino RJ. Medical rehabilitation length of stay and outcomes for persons with traumatic spinal cord injury: 1990-1997. Arch Phys Med Rehabil 1999;80:1457-63.

22. Afsar SI, Sarifakioglu B, Yalbuzdag SA, Saracgil Cosar SN. An unresolved relationship: the relationship between lesion severity and neurogenic bladder in patients with spinal cord injury. J Spinal Cord Med 2016;39:93-8.

23. Skilton MR, Moulin P, Serusclat A, Nony P, Bonnet F. A comparison of the NCEP-ATPIII, IDF and AHA/ NHLBI metabolic syndrome definitions with relation to early carotid atherosclerosis in subjects with hypercholesterolemia or at risk of CVD: evidence for sexspecific differences. Atherosclerosis 2007;190:416-22.

24. Yahiro AM, Wingo BC, Kunwor S, Parton J, Ellis AC. Classification of obesity, cardiometabolic risk, and metabolic syndrome in adults with spinal cord injury. J Spinal Cord Med 2020;43:485-96.

25. Hirode G, Wong RJ. Trends in the prevalence of metabolic syndrome in the United States, 2011-2016. JAMA 2020;323:2526-8.

26. Gater DR Jr, Farkas GJ, Berg AS, Castillo C. Prevalence of metabolic syndrome in veterans with spinal cord injury. J Spinal Cord Med 2019;42:86-93.

27. Examination Committee of Criteria for 'Obesity Disease' in Japan; Japan Society for the Study of Obesity. New criteria for 'obesity disease' in Japan. Circ J 2002;66:987-92.

28. Anuurad E, Shiwaku K, Nogi A, Kitajima K, Enkhmaa $\mathrm{B}$, Shimono $\mathrm{K}$, et al. The new BMI criteria for asians by the regional office for the western pacific region of WHO are suitable for screening of overweight to prevent metabolic syndrome in elder Japanese workers. J Occup Health 2003;45:335-43.

29. Bluvshtein V, Korczyn AD, Pinhas I, Vered Y, Gelernter I, Catz A. Insulin resistance in tetraplegia but not in mid-thoracic paraplegia: is the mid-thoracic spi- nal cord involved in glucose regulation? Spinal Cord 2011;49:648-52.

30. Gater DR Jr. Obesity after spinal cord injury. Phys Med Rehabil Clin N Am 2007;18:333-51.

31. Gupta N, White KT, Sandford PR. Body mass index in spinal cord injury: a retrospective study. Spinal Cord 2006;44:92-4.

32. Weaver FM, Collins EG, Kurichi J, Miskevics S, Smith B, Rajan S, et al. Prevalence of obesity and high blood pressure in veterans with spinal cord injuries and disorders: a retrospective review. Am J Phys Med Rehabil 2007;86:22-9.

33. Krassioukov A, Claydon VE. The clinical problems in cardiovascular control following spinal cord injury: an overview. Prog Brain Res 2006;152:223-9.

34. Myers J, Lee M, Kiratli J. Cardiovascular disease in spinal cord injury: an overview of prevalence, risk, evaluation, and management. Am J Phys Med Rehabil 2007;86:142-52.

35. Pischon T, Boeing H, Hoffmann K, Bergmann M, Schulze MB, Overvad K, et al. General and abdominal adiposity and risk of death in Europe. N Engl J Med 2008;359:2105-20.

36. Cerhan JR, Moore SC, Jacobs EJ, Kitahara CM, Rosenberg PS, Adami HO, et al. A pooled analysis of waist circumference and mortality in 650,000 adults. Mayo Clin Proc 2014;89:335-45.

37. Song X, Jousilahti P, Stehouwer CD, Soderberg S, Onat A, Laatikainen T, et al. Comparison of various surrogate obesity indicators as predictors of cardiovascular mortality in four European populations. Eur J Clin Nutr 2013;67:1298-302.

38. Zhang C, Rexrode KM, van Dam RM, Li TY, Hu FB. Abdominal obesity and the risk of all-cause, cardiovascular, and cancer mortality: sixteen years of followup in US women. Circulation 2008;117:1658-67.

39. Laughton GE, Buchholz AC, Martin Ginis KA, Goy RE; SHAPE SCI Research Group. Lowering body mass index cutoffs better identifies obese persons with spinal cord injury. Spinal Cord 2009;47:757-62.

40. Coppini LZ, Waitzberg DL, Campos AC. Limitations and validation of bioelectrical impedance analysis in morbidly obese patients. Curr Opin Clin Nutr Metab Care 2005;8:329-32. 